\title{
Transalpina
}

TRANSALPINA Études italiennes

$20 \mid 2017$

Edmondo De Amicis. Littérature et société

\section{Luca Bani et Yannick Gouchan, La figura del fanciullo nell'opera di D'Annunzio, di Pascoli e dei crepuscolari}

\section{Mariella Colin}

\section{(2) OpenEdition}

\section{Journals}

Édition électronique

URL : http://journals.openedition.org/transalpina/414

DOI : $10.4000 /$ transalpina.414

ISSN : 2534-5184

Éditeur

Presses universitaires de Caen

\section{Édition imprimée}

Date de publication : 1 septembre 2017

Pagination : 275-278

ISBN : 978-2-84133-857-3

ISSN : $1278-334 \mathrm{X}$

\section{Référence électronique}

Mariella Colin, « Luca Bani et Yannick Gouchan, La figura del fanciullo nell'opera di D’Annunzio, di Pascoli e dei crepuscolari », Transalpina [En ligne], 20 | 2017, mis en ligne le 19 décembre 2019, consulté le 07 novembre 2020. URL : http://journals.openedition.org/transalpina/414; DOI : https://doi.org/10.4000/ transalpina. 414 
perception d'une absence associée à la nostalgie d'un passé glorieux, que la langue italienne n'a nullement compensé (Alfredo Luzi); quant aux Italiens, d'après le sarcasme amer et désespéré du Discorso sopra lo stato presente dei costumi degl'italiani, le cynisme est le trait qui caractérise le mieux ce peuple qui a perdu son ancienne grandeur (Fulvio Senardi).

Viennent alors les mythes léopardiens, créations poétiques et philosophiques de l'auteur des Canti. Premier d'entre eux, le mythe de la lune; en particulier dans Spavento notturno et Canto notturno di un pastore errante d'Asia, où l'astre est tour à tour lumière (point de référence, guide), visage (spectatrice des vicissitudes terrestres), divinité omnisciente par sa position; mais c'est une divinité déchue et absente (Antonella Del Gatto). Les ruines ont une double fonction, critique et gnoséologique: symbole par excellence de la grandeur de l'Antiquité, elles évoquent un temps prestigieux mais irrécupérable dans All'Italia, mais sont la preuve de la fragilité des choses et des hommes devant la Nature dans La Ginestra (Patrizia Landi). Dans celle-ci, le poète crée un mythe moderne de la métamorphose, lorsque ce ne sont plus les figures mythologiques qui se transforment, mais le poète lui-même, quand le genêt devient le témoin de la disparition de la civilisation (David Jérôme) et que la «noble nature» qui se dresse face au mal qui pèse sur la condition humaine devient une figure mythique à forte valeur autobiographique (Giuseppe Antonio Camerino). C'est ainsi que Leopardi peut devenir lui-même un mythe, lorsque les fondamentaux de sa pensée prennent une valeur mythique aux yeux du lecteur (Fabrice De Poli), ou bien quand des philosophes tels que Gentile, Croce et Gramsci accréditent l'idée d'un pessimisme philosophique qui provoquerait l'optimisme du cœur ou du moins de l'action (Stéphanie Lanfranchi).

Mariella Colin

\section{Luca Bani et Yannick Gouchan, La figura del fanciullo nell'opera di D’Annunzio, di Pascoli e dei crepuscolari, Milan, Cisalpino, 2015, 284 p.}

Avec cet essai à quatre mains, Luca Bani et Yannick Gouchan se penchent sur un domaine qui n'avait pas encore été étudié en profondeur par la critique: celui de la construction et de la signification de la figure de l'enfant dans la poésie italienne contemporaine, lorsque ce thème devient central dans l'imaginaire littéraire. Il servira aux deux critiques de fil conducteur pour interpréter un important corpus d'œuvres entre la fin du XIX et le début du XX ${ }^{\mathrm{e}}$ siècle, unissant les deux poètes majeurs que sont $\mathrm{D}^{\prime}$ Annunzio et Pascoli au groupe des poètes «crépusculaires». 
L'ouvrage s'ouvre par un chapitre introductif de Luca Bani, qui retrace l'évolution de la représentation enfantine depuis l'Antiquité jusqu'à la fin $\mathrm{du} \mathrm{XIX}^{\mathrm{e}}$ siècle, dans une synthèse attentive aux étapes principales qui la jalonnent. L'enfant "fuori del tempo» de la civilisation grecque et latine devient un être marqué par la faute dans les textes chrétiens, avant de connaître un nouveau statut au XVIII ${ }^{e}$ siècle (notamment avec Rousseau), puis à l'époque du romantisme allemand (lorsque l'enfant atteint une condition ontologique de perfection et de pureté absolues). Naît ainsi le culte de l'enfance, dont Dickens, qui fait de ses personnages enfantins (Oliver Twist, David Copperfield, Nicolas Nickleby, Little Dorrit) les protagonistes de ses romans, sera le premier grand représentant en littérature. Viendra ensuite la floraison européenne de la nouvelle catégorie du Bildungsroman, avant la représentation vériste de l'enfant travailleur, puis la remise en question de l'idéalisation enfantine par des écrivains tels qu'Henri James (Le Tour d'écrou) et Thomas Mann (La mort à Venise).

Dans le deuxième chapitre, le thème de l'enfant sert de fil conducteur à L. Bani pour une exploration des images et des motifs par lesquels il s'inscrit dans l'œuvre de D'Annunzio. Le traitement de la figure enfantine est marqué par une opposition irréductible entre la prose et la poésie de l'auteur: l'une dramatise et humilie la condition enfantine, l'autre la magnifie. Dans la littérature narrative du pescarese, les recueils de nouvelles mettent en scène la nature sauvage et la société primitive des Abruzzes d'après le canon du naturalisme: dans Terra vergine, les enfants sont décrits comme des êtres monstrueux, plus proches des bêtes que des humains, selon le goût pour l'horrible et le répugnant qui caractérise D'Annunzio vériste; dans $L e$ novelle della Pescara, ce sont souvent des êtres misérables, physiquement et moralement, dont l'enfance est sans espoir et la vie sans futur. Les romans en revanche mettent en scène l'enfant de la bourgeoisie, et en font un élément topique pour refléter l'esthétique du décadentisme et signifier le pessimisme éthique et existentiel de l'écrivain. Dans Il Piacere, à côté de l'enfance populaire apparaît l'enfance bourgeoise, fragile et maladive; dans le Trionfo della morte, les personnages enfantins préfigurent l'«inettitudine a vivere» de l'artiste bourgeois, et sont des messagers de la mort qui attend le protagoniste. Il en va tout autrement dans la poésie, où le motif de l'enfance et la figure de l'enfant vont être au cœur de l'évolution de la poétique dannunzienne: dans Canto novo (livre III), l'enfant est un être "primitif", mais fier et doté d'une force primordiale, alter ego de D'Annunzio rêvant d'une nature panique et d'une terre vierge; dans le Poema paradisiaco apparaît le thème nouveau de l'innocence liée à l'enfance et au retour à l'espace domestique des années juvéniles, et dans la figure du puer se réalise le rêve de la régénération du poète. En revanche, dans le recueil 
suivant (Maia) commence le parcours qui aboutira à une vision mythique de la Grèce où se fondent enfance de l'artiste et enfance du monde; un parcours qui sera couronné ensuite dans Alcyone par le triomphe de l'esprit dionysiaque, s'incarnant dans l'enfant-dieu Hermès. Musicien et archer, poète et guerrier, hypotypose de la nature et de l'art, «nel fanciullo alcyonio è possibile rintracciare l'essenza più pura e sincera della poetica dannunziana" (p. 123), une poétique qui sera reprise dans le livre III des Laudi.

Dans les chapitres suivants, Yannick Gouchan poursuit la même investigation chez les poètes du début du $\mathrm{XX}^{\mathrm{e}}$ siècle. Pascoli s'impose d'emblée comme le "poeta del Fanciullino", tant son esthétique, proche du symbolisme européen, apparaît rattachée à la métaphore du puer ut poeta, possédant la capacité de s'émerveiller et de s'émouvoir qui est à l'origine de la création artistique; le poète qui lui ressemble est celui qui saura décrire le monde et exprimer les sentiments. Si l'enfant métaphorique est la figure centrale de la poétique pascolienne, les personnages enfantins qui peuplent ses compositions sont porteurs d'une forte composante autobiographique, liée à la tragédie familiale vécue par le poète; Myricae avant tout, mais aussi Primi poemetti, Nuovi poemetti et Canti di Castelvecchio sont peuplés de figures pathétiques d'enfants confrontés à la misère et à la mort, la leur ou celle de leurs parents. Les mêmes racines autobiographiques sont à l'origine d'autres motifs soulignés par Y. Gouchan, comme les métaphores ornithologiques des hirondelles ou du nid (le foyer familial détruit par l'assassinat du père, que le poète a voulu ensuite reconstruire avec ses jeunes sœurs) et les métaphores florales (qui représentent l'Eden de l'enfance, mais aussi le sort des jeunes êtres fauchés par la mort comme des fleurs pas encore écloses). Une réflexion plus générale sur la condition humaine est celle que le «fanciullo etico» peut donner à l'adulte, en réalisant les idéaux de la solidarité et de la fraternité comme dans les deux apologues emblématiques de I due fanciulli et I due orfani; mais cette identification avec l'enfant idéal comporte également une connotation régressive, signifiant le refus de l'âge adulte, car il est une nouvelle manière pour le poète de se placer en dehors du temps historique et de la société. Viennent en dernier lieu les élèves-lecteurs des anthologies de littérature latine (Lyra romana, Epos) et italienne (Sul limitare, Fior da fiore) que Pascoli composa pour les élèves des écoles secondaires. Une mission pédagogique également influencée par la poétique du Fanciullino, qui voit dans ces destinataires des alter ego capables d'entendre la véritable poésie parce qu'encore proches des privilèges de l'enfance.

Le dernier chapitre porte sur «la condition crépusculaire de l'enfant»: la disposition esthétique et existentielle d'une pléiade de poètes relevant du même courant (Govoni, Moretti, Corazzini, Palazzeschi, Gozzano, Civinini, 
Vallini...), chez lesquels la figure enfantine est plurielle, mais toujours nécessaire pour interpréter le présent des adultes. Il ne s'agit plus de l'enfant divin de Pascoli, ni de l'enfant sublime de D'Annunzio, mais d'un enfant réel, une créature souvent en marge du monde des adultes, à laquelle le poète s'identifie dans sa recherche d'un refuge; non plus un enfant-poète mais un poète-enfant souvent enclin à la tristesse, à la mélancolie et aux larmes ("Io non sono che un piccolo fanciullo che piange», écrit Corazzini); un enfant souvent malade qui se complaît dans son état de faiblesse, un enfant inerte et fragile dont la condition est exempte de pathétisme. Dans ce répertoire d'images pour la création poétique, dont Y. Gouchan dresse ici un riche inventaire, figurent d'autres motifs censés offrir un refuge contre les déceptions du présent, comme le chronotope de la maison de l'enfance (Gozzano, Vallini) ou celui de l'école primaire (Moretti). Mais ces rêveries d'enfance débouchent inévitablement pour les poètes crépusculaires sur le constat d'un impossible retour aux joies et à la liberté de la condition enfantine encore ouverte sur le futur.

Mariella CoLIN 